Loading

The Journal of the Canadian Game Studies Association

\title{
Making Ourselves Visible: Mobilizing Micro-Autoethnography in the Study of Self-Representation and Interface Affordances
}

\section{Victoria McArthur}

Volume 12, Number 19, Winter-Spring 2019

URI: https://id.erudit.org/iderudit/1058319ar

DOI: https://doi.org/10.7202/1058319ar

See table of contents

Publisher(s)

Canadian Game Studies Association

ISSN

1923-2691 (digital)

Explore this journal

Cite this article

McArthur, V. (2019). Making Ourselves Visible: Mobilizing

Micro-Autoethnography in the Study of Self-Representation and Interface

Affordances. Loading, 12(19). https://doi.org/10.7202/1058319ar

\section{Article abstract}

Avatar customization and self-representation in games has been widely studied. In this paper, I propose the use of micro-autoethnography as a complementary methodology in such studies. I propose such an approach, theoretically and methodologically informed by Actor-Network Theory, as a way for researchers to situate themselves within their own studies of identity and play in games. I present a micro-autoethnographic study in which I, the researcher, attempt to create the same avatar in eight different Character Creation Interfaces (CCIs), otherwise known as a "trans-ludic" avatar. Implications for a micro-autoethnographic approach to avatar and identity research are discussed.
This document is protected by copyright law. Use of the services of Erudit (including reproduction) is subject to its terms and conditions, which can be viewed online.

https://apropos.erudit.org/en/users/policy-on-use 


\title{
Making Ourselves Visible: Mobilizing Micro- Autoethnography in the Study of Self-Representation and Interface Affordances
}

\author{
Victoria McArthur \\ Carleton University \\ victoria.mcarthur@carleton.ca
}

\begin{abstract}
Avatar customization and self-representation in games has been widely studied. What is often missing from many games papers is a positioning of the researcher(s) in relation to their work. In this paper, I propose the use of micro-autoethnography as a complementary methodology in such studies. I propose such an approach, theoretically and methodologically informed by ActorNetwork Theory, as a way for researchers to situate themselves within their own studies of identity and play in games. I present a micro-autoethnographic study in which I, the researcher, attempt to create the same avatar in eight different Character Creation Interfaces (CCIs), otherwise known as a "trans-ludic" avatar. The resultant avatars are similar, but not the same the result of negotiating differing interface affordances and game world aesthetics. The differences between each interface are systematically narrativised and analyzed via this microautoethnographic exercise. I envision this method being taken up as a complementary approach to identity study in games where avatar customization is being studied. Further, the exercise may be useful as an instructional tool in game design programs in order to guide students in making informed choices about CCI design.
\end{abstract}

\section{Author Keywords}

Avatars; Self-representation; Autoethnography; Micro-ethnography; Actor-Network Theory.

\section{Introduction}

Avatar customization is a feature offered in many digital games and social virtual worlds. This feature is commonly presented via a graphical user interface, referred to herein as a Character Creation Interface or CCI. CCIs present various customization options to players via interface widgets (e.g., sliders for avatar height, colour palettes for avatar hair colour). The affordances the quality and quantity of customization options, as well as the way that they are presented to the user - differs greatly from game to game. Some of these differences are somewhat determined by the aesthetics and genre of the game or virtual world. In the literature, the term "high fidelity" has been presented as a way to classify CCIs that are highly complex and 
presumably afford a diverse set of customization options (Ducheneaut, Wen, Yee, \& Wadley, 2009).

Studies of CCIs and self-representation in games are numerous, and include a wide variety of methodologies, including surveys (Ducheneaut, et al., 2009; Kafai, Fields, \& Cook, 2010b), online ethnographic methods (Kafai, Fields, \& Cook, 2010a; Nardi, 2010; Carman Neustaedter \& Fedorovskaya, 2008), lab-based observational studies (Bergstrom, Jenson, \& de Castell, 2012; McArthur \& Jenson, 2015), and interface analysis (Consalvo, 2003b; McArthur, Jenson, \& Teather, 2015; Pace, 2008; Pace, Houssian, \& McArthur, 2009). In each of these studies, one or more games/virtual worlds are identified and subsequently analyzed for the purpose of advancing theories on self-representation and identity in games.

In this paper, I propose the use of micro-autoethnography as a complementary methodology in such studies. Although it is uncommon to author a research paper in the first person, even where there is only a single author, the research and data presented herein is the result of an autoethnographic approach which necessitates a less-formal perspective in order to integrate the data into the analysis. I argue that an autoethnographic approach presents researchers with the methodological means to situate themselves within their research, making visible the ways in which their own biases, past experiences, and position came to shape their hypotheses and analyses in research on self-representation in games.

\section{Related Work}

Although avatar customization has been widely studied, much of the related work in this area is anthropocentric, focusing on the kinds of "bodies" and identities users craft in MassivelyMultiplayer Online Games (MMOGs) and social virtual worlds (e.g., see Bessière, Seay, \& Kiesler, 2007; Ducheneaut, et al., 2009; Kafai, et al., 2010b; Neustaedter \& Fedorovskaya, 2009). In many of these papers, the authors focus on the choices users make in customizing their avatars and how identity fidelity (how "accurately" they represent themselves via avatar appearance) contributes to an understanding of the pragmatics of self-representation online. For example, papers by Ducheneaut et al. (2009) and Kafai et al. (2010b) utilize survey and online interviews respectively to collect data on the self-representational practices of users in MMOGs. Using the self-reported data collected from their participants, both papers report that users' avatar customization preferences could be grouped under one of three or four trends (e.g., the desire to create a representative avatar, the desire to create a new online identity, or the desire to create an avatar that follows a popular trend in the virtual world). In these papers, and others like them, avatar creation interfaces are presented as tools that help users create an online identity.

Almost all MMOGs limit players to two sexes, yet text-based environments allowed users to move beyond this heteronormative binary (Sundén, 2003). Present-day MMOGs tend to lock users into the male/female binary, leaving little room for "high-fidelity" in the GLBTQ population. Sundén acknowledges how the design of avatars, both in how they are "constructed" 
and how they are programmed to perform in-game, puts many users in tension with the heteronormative design of their second selves (Sundén, 2009, p. 3).

The research of Kafai et al. (2010a) and Consalvo (2003a) highlights issues of ethnic representation in MMOGs. For example, in studying the tween virtual world Whyville, Kafai et al. (2010a) note the general lack of non-white faces available to players. Consalvo's (2003a) work on the character creation interface in The Sims reveals that the interface defaulted to a lightskinned, middle-aged male every time, despite the fact that it is possible to create Sims that are not white and/or are not male.

Thus, while many CCIs offer an extensive range of customization options, research has shown that, through affordances, CCIs have the potential to represent socially exclusive values (Kolko, 1999; Pace, et al., 2009). Both the quality and quantity of customization options present in a given CCI determines how significant identity categories, such as gender and ethnicity, may be represented via avatar bodies (e.g., see Consalvo, 2003b; Dietrich, 2013; Downs \& Smith, 2005; Higgin, 2009, forthcoming; Kafai, et al., 2010a; Leonard, 2006; Pace, 2008; Pace, et al., 2009). In each of these papers, researchers looked to interface analysis as a means to study the effects of affordances on social inclusion, but did not study user interactions with the interface.

\section{Theoretical Framing}

\section{Cultural Rhetoric}

Salen \& Zimmerman (2004) use the term "cultural rhetoric" to describe games as ideological systems based on offline ideologies. Bogost (2010) goes one step further than Salen and Zimmerman in suggesting that video games are capable of a new kind of rhetorical expression, one he refers to as "procedural rhetoric." For Bogost, procedural rhetoric is an argument expressed through computer code, where a game's rules and mechanics convey an argument about the state of things - an argument that must be read by actually playing the game. The ability to read a procedural argument requires a specialized literacy, one Bogost refers to as procedural literacy: "...videogame players develop procedural literacy through interacting with the abstract models of specific real or imagined processes presented in the games they play. Videogames teach biased perspectives about how things work. And the way they teach such perspectives is through procedural rhetorics, which players 'read' though direct engagement and criticism" (p. 260). Thus, it is clear that ideologies can be encoded, by programming or by design, and that such ideologies have not gone unnoticed.

In the context of game character design, these ideologies are often seen as heteronormative and Caucasian, based on the ways in which player characters and non-player characters are represented in games (Consalvo, 2003a; Higgin, 2009; Leonard, 2006; Pace, et al., 2009). While the idealized player base may not take issue with the representation of minorities in games, many other player communities, in particular the GLBTQ community, have adopted what Sundén (2009) refers to as transgressive play, which she describes as "play against the 'ideal' or 
'implied' player of the game, of playing the game and bending the rules in ways not anticipated by design...as innovation and, possibly, subversion, of finding, exploring and exploiting loopholes in the game fabric" (p. 2)

\section{Actor-Networks}

Mobilizing Actor-Network Theory (Callon, 1997; Latour, 1987, 1992, 2005; Law, 1992, 2004)

as a theoretical lens allows us as researchers to isolate the actors (human and non-human) who play a role in identity construction and performance in digital games and social virtual worlds. The most significant contribution yielded by such a theoretical shift is that the non-human actors who shape our in-game experiences, in particular our self-representational practices, are not only made visible, but are understood as having an effect on these practices. As Latour (2005) suggests, by "render[ing] the social world as flat as possible" (p. 16), we are able to view all nodes in the network of a given social phenomena - even those nodes which have previously been overlooked or rendered invisible by the research. Much of the aforementioned literature has privileged certain actors over others, especially human actors and their agency within the network. Character creation interfaces have all but been ignored as actors, even though they contribute a great deal to self-representation in terms of mediation and affordances.

The somewhat controversial attribution of agency to non-humans in ANT grants agency to the interface as an actor, rather than simply acknowledging it as a tool which the user engages with when making choices about avatar customization (Latour, 2005). An authentic ANT approach also makes the researcher and their own self-representational practices more visible in the literature. The researcher's own biases, assumptions, training, and experiences shape the truths that otherwise appear to be "revealed" in doing research (Law, 2004).

What is often missing from many games papers is a positioning of the researcher(s) in relation to their work. Often times, researchers will disclose having prior experience playing within the games that they study, however, as noted by Taylor (2008), this information is never shared again within the context of the study design or resultant analyses. This information seems to serve the purpose of positioning the researcher's familiarity as sufficiently authoritative to make the contributions outlined in their studies. However, the researcher is the actor who proposes the theories, names the actors, and traces the network, making its nodes visible - or invisible. Law (2004) describes this process as ontological politics. As Law suggests, "[i]n an ontological politics we might hope, instead, to make some realities realer, others less so" (Law, 2004, p. 67). Even though unintentional, the researcher shapes the network, chooses which nodes to elevate to the status of 'actor' and which actors are worth studying.

The method outlined herein, and the impetus for its use, is motivated by this issue. As Taylor (2008) notes, we, as researchers must "...begin to see, and account for, [our] own embodied experiences playing in particular discursive and material contexts as fundamental to how [we] arrive at knowledge about a game" (p. 2). 


\section{Trans-Ludic Identities}

One such example involves the longitudinal, fully immersive online ethnography of Celia Pearce, in her study of the Uru diaspora (Pearce \& Artemesia, 2008, 2009a). The term transludic identity originated with this work, wherein Pearce proposed it as a way to conceptualize purposeful identity continuity by a single user across multiple game spaces (Pearce \& Artemesia, 2008, 2009a). For Pearce, the trans-ludic identity became evident in her ethnographic study of the Uru diaspora. Following the abrupt closure of the MMOG Uru: Ages Beyond Myst, players of the game migrated to other game spaces and re-created their Uru avatars and other aspects of the game world in an attempt to maintain their ludic space. Pearce noted that the disparity in affordances between the different gaming spaces had a direct impact on the degree to which players can express trans-ludic identities (Pearce \& Artemesia, 2009a). While Pearce was the first to theorize about the trans-ludic identity, identity continuity in virtual worlds has been studied by others in terms of naming practices (Lawson \& Murray, 2014), behavioural practices (Neustaedter \& Fedorovskaya, 2009), and avatar customization across multiple games (Taylor, McArthur, \& Jenson, 2012).

As noted by Pearce, it is often not possible to create the same avatar across multiple game spaces since interface affordances and aesthetics vary from game to game (Pearce \& Artemesia, 2009a). Pearce describes the creative challenge of creating her trans-ludic avatar, Artemesia, and how she negotiated these challenges:

In each case, the general appearance includes variants of red/titian/copper hair, a fair complexion, and one of a number of hairdos that attempted to approximate either past or current hairstyles I've had in real life. As each virtual world has different set of affordances for avatar creation... recreating the same characters across game worlds turns out to be a creative challenge. How can you capture the essence of a character when faced with a fundamentally different mechanism for self-portraiture?

Pearce \& Artemesia, p. 197 - 198 (2009a)

While the term "trans-ludic" is not widely present in the literature which theorizes selfrepresentational practices, I would propose that the interest in, and prevalence of, "realistic" and "idealized" avatars in the literature is indicative of an awareness of trans-ludic identities, even if they have more in common with their creator than each other (Ducheneaut, et al., 2009; Jin, 2009, 2010; Kafai, et al., 2010b; Neustaedter \& Fedorovskaya, 2008). If one were to shift one's focus from the user (anthropocentric) to the avatars themselves, their trans-ludic qualities are made visible. This is not to say that human actors are insignificant, but that their significance has a tendency to render other nodes in the network invisible, which can produce limited theories on self-representation in games and virtual worlds. In other words, the focus on anthropocentricism has obscured our understanding of self-representational practices. 


\section{Method \& Methodology}

The method presented herein is a combination of autoethnography (Ellingson \& Ellis, 2008; Ellis, 2004; Maréchal, 2010; Reed-Danahay, 1997; Spry, 2001) and microethnography (Giddings, 2009; Giddings \& Kennedy, 2008; Streeck \& Mehus, 2005), which I will refer to here as a micro-autoethnography. For the study, I created eight trans-ludic avatars, one per game, in the following games: World of Warcraft, EVE Online, Saints Row IV, Jam City Rollergirls, Skyrim, RuneScape 3, Rift, and the Mii Creator.

\section{Methodology}

Autoethnography is a qualitative research method - a form of self-reflection in which the researcher's own personal experiences are documented and reflected upon in order to connect the researcher's own thoughts and ideas to the wider cultural understandings of a given phenomenon. Ellis (2004) defines it as "research, writing, story, and method that connect the autobiographical and personal to the cultural, social, and political" (Ellis, 2004, p. 48). Therefore, unlike other research methods, autoethnography offers a chance for researchers to embrace their subjective experiences, rather than attempting to limit subjectivity in the interest of objectivity. Autoethnographic approaches have been adopted in game studies and are particularly prevalent in qualitative studies of MMOGs, wherein the researcher joins a guild and reports on their own play experiences within her network of peers and friends (Chen, 2009; Nardi, 2010; Pearce \& Artemesia, 2009b; Taylor, 2006).

While the highly subjective nature of autoethnographies makes it difficult to extrapolate from these studies to larger populations of MMOG players (Taylor, et al., 2012), when used in concert with other methods, autoethography not only presents the researcher with an opportunity to methodologically disclose their own subjectivity, but also to present additional data that may provide an outsider with a more intimate account of the subject of study. Boellstorff et al. (2012) describe autoethnography (as conducted in the virtual world) as a method that may potentially "yield new insights and discoveries", but caution that autoethnography has also been used to mask a lack of method or experimental design (p. 44). Thus, in conducting an autoethnography, the researcher must take great care in being consciously disciplined in their approach.

Microethnography, originating with the work of Smith and Geoffrey (1968), was proposed as a means to deploy ethnographic methods to study the "complexities of an urban classroom" (p. 3). In this case, "micro" referred to the size of the cultural phenomena they examined, rather than the methods themselves. Contemporary microethnography refers to the recording of short events, and utilizing interactionist modes of analysis (Streeck \& Mehus, 2005). Capturing the event on video allows researchers to focus on specific micro-events that reveal the "foundations of social organization, culture and interaction at the micro level of the moment-by-moment development of human activities" (p. 381). 
More recently, in the context of game studies, microethnography has been adapted to game studies by game scholars Giddings and Kennedy (Giddings, 2009; Giddings \& Kennedy, 2008), who outline their method as follows:

1. Both the television screen and player(s) are captured on video. These recordings are then synchronized so that they can be viewed simultaneously.

2. Player speech and key game events are then transcribed.

3. Short sections are then selected for in-depth analysis.

Video data is then entered into behavioural analysis software (e.g., Morae, Noldus) and analyzed using interactionist modes of analysis (Giddings, 2009; Giddings \& Kennedy, 2008). It is worth noting here that Giddings and Kennedy's (2008) own study of Lego Star Wars, was essentially a micro-autoethnography of the two authors as they learned to play the game and video recorded their play. The researchers' own animated commentary highlighted the complexity of the tiny processes that occurred and allowed them to "gain some purchase on the intangibilities of gameplay" (p. 3).

In order to provide an authentic, reflective account of my own experiences creating avatars, the think aloud protocol (Lewis, 1982) was used. This protocol is an explicit method of user experience research in which participants perform a verbal, real-time self-report on their experiences while using a particular interactive artefact or, in this case, video game. The think aloud method differs from the talk aloud method, such that the latter insists that they describe their actions without reflecting on them. The think aloud method is often valued for its subjectivity, while the talk aloud method is valued for its objectivity (Ericsson \& Simon, 1993).

Each avatar creation session was timed in seconds. In the analysis section which follows, I have also provided reflections on my own prior experiences with each of the games. This, I hope, will provide researchers with a novel method for disclosing their own biases and positioning themselves within their research.

\section{Apparatus}

The experiment was run on an Alienware M14X laptop running the Windows 7 Home Premium 64 bit operating system. All PC games were played on this laptop, and the two console-based interfaces (Jam City Rollergirls, Mii Creator) were played on the WiiU. In all cases, the AVerMedia C875 Live Gamer Portable (LGP) HD Game Capture Video Device was used to screen capture the games. XSplit Gamecaster software was used to simultaneously record input from the games and video from the laptop's built-in webcam. All games, including the console output, was displayed on the laptop's 14 inch screen and the webcam was directed towards the participant. For the Mii Creator interface, I used the WiiU Game Pad to interact with the console. 


\section{Results: My Trans-ludic Selves}

Prior to the start of the exercise, I reflected upon my own prior experiences playing each of the games and documented these details in an ethnographer's journal. In these notes, I started by thinking back to when I began playing each game, where and when I would often play, how I came to play that game, and who I played with. In addition, I also spent some time visually analyzing all of my prior avatars (mains and alts), noting any similarities and differences with regard to visual self-representation both within and across ludic spaces.

These details make my own biases much more visible and have previously contributed to my own analyses on self-representational practices. For example, when reflecting upon my first play experiences in World of Warcraft, I recall how I came to play the game with my partner, and how my choice to play an undead (Horde) character had an impact on my partner's own selfrepresentation in game:

"I first began playing World of Warcraft in 2007 with my partner. We had both purchased the game with the intention of playing together. As fate would have it, I installed the game first and was the first to create a character. After spending a fair amount of time previewing all of the game's playable races, I decided to play as an undead female warrior... When my partner joined me in Azeroth the next day, he created an undead warlock... In later discussions, he would reveal to me that he would have preferred to have played for the Alliance, not the Horde, based on his experiences playing the other Warcraft games (games that I had no experience playing). Although I was quite attached to my main avatar, I realized that, had we created avatars at the same time, I could have just as easily ended up creating an avatar from the opposite faction, most likely a human warrior. While this may not be a universal experience, I was amazed at how the desire to play together had such an impact on avatar customization. Faction, for co-situated players, must be negotiated at the onset of character creation."

This effect is noted in my own study of Rift players (McArthur \& Jenson, 2015) where players in the study who wished to play together had to choose the same faction (Guardian or Defiant). This subsequently had an impact on the player's self-representation in-game - a significant factor contributing to the player's self-representational practices. When technological systems are opened up to a wider analysis via a theoretical framework such as ANT - or any other that takesup the sociotechnical - similar effects can be anticipated in online identity work. Yet such factors are typically absent in most theoretical work on self-representational practices.

"Recalling my own gaming experiences, there are a few fundamental similarities between all of the avatars I have created to date. Looking at my various mains and alts, I notice that I nearly always play as a female avatar. In real life, I naturally have red hair, although I have also experimented quite a bit with alternative hair colours (e.g., pinks, purples, greens, blues, etc.). Whenever a character creation interface presents these nontraditional hair colour options, I tend to gravitate towards them. When these options have 
been limited to one playable race or faction, this has sometimes influenced my choice of race or faction...Reflecting upon my own self-representational practices, it is clear that I desire a degree of fidelity when customizing my avatars."

As stated previously, these notes also included details on all of my previous avatars in these gaming spaces. Like Pearce, I found that my own avatars seemed to follow a trend of being somewhat representative, albeit somewhat idealized, versions of myself. The above paragraph is, perhaps, a bit lengthy, but demonstrates that, reflectively, I had noticed a pattern in my own selfrepresentational practices, a trend that is not uncommon in trans-ludic play where avatar customization is available (Ducheneaut, et al., 2009; Jin, 2009, 2010; Kafai, et al., 2010b; Neustaedter \& Fedorovskaya, 2008). Such mappings have been noted, for instance in papers by Ducheneaut et al. (2009) and Kafai et al. (2010b), where customization trends were noted among participants in each of these studies. However, it is important to note that not all players adopt the same strategy across game spaces (Taylor, et al., 2012), and that self-representation can also be affected by spontaneous discoveries made within the CCI during avatar customization.

Once I had completed preliminary notes and analyses on my past experiences playing and customizing avatars in each of the game spaces, I then proceeded to create a trans-ludic, representative avatar in each game: World of Warcraft, EVE Online, Saints Row IV, Jam City Rollergirls, Skyrim, RuneScape 3, Rift, and the Mii Creator. Resultant avatars are shown in Figure 1. Subsequent video footage was then transcribed and analyzed for UI selection and manipulation events. In addition, a reflective narrative on the experience was also recorded by hand and included in the analysis.

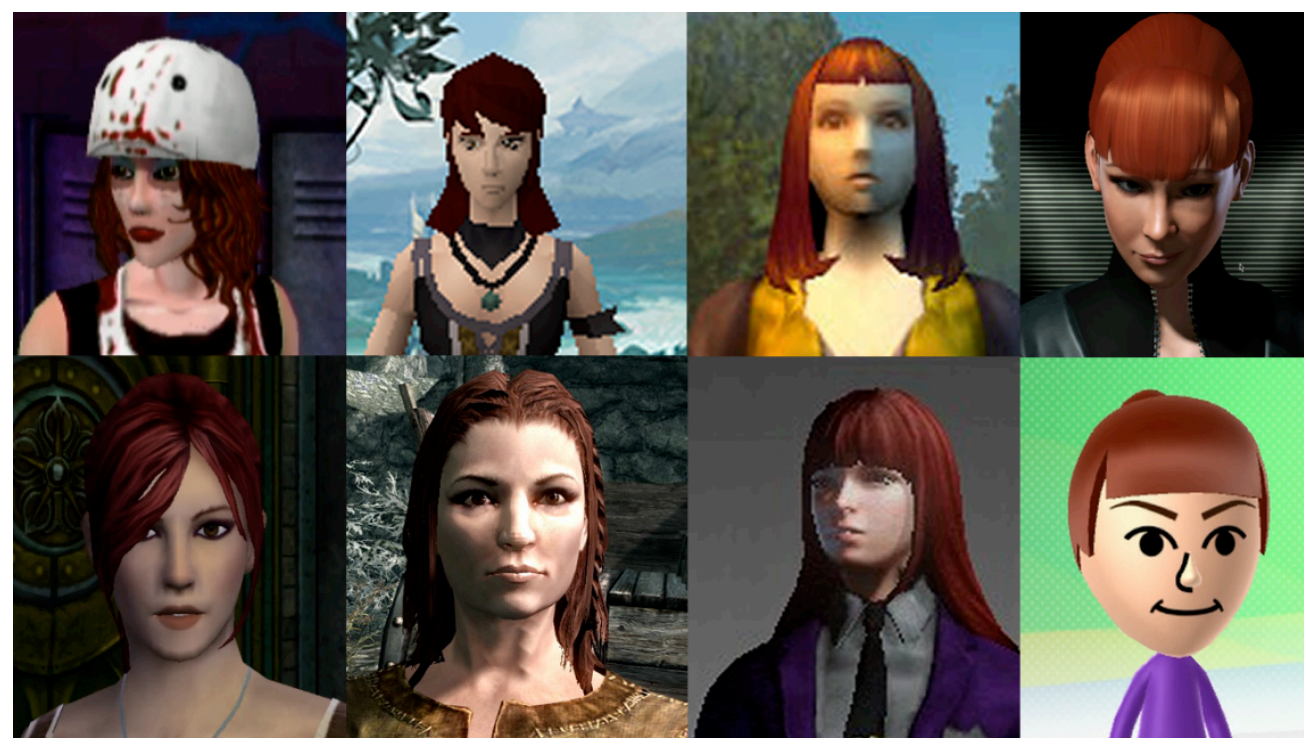

Figure 1: Eight avatars created in the micro-autoethnographic task.

It is important to note here that, although this paper is theoretically framed by a discussion of social inclusion in avatar customization, I have still chosen to create a representative (white) 
avatar. This decision is informed by Lisa Nakamura's (2002) discussions on identity tourism online, in which she describes the problematic affordance offered by virtual spaces, allowing one to appropriate another culture or identity online. Thus, I have chosen to create only representative avatars in this paper, not only to situate myself within the research, but also to avoid making problematic claims about identity affordances to which I have no claim.

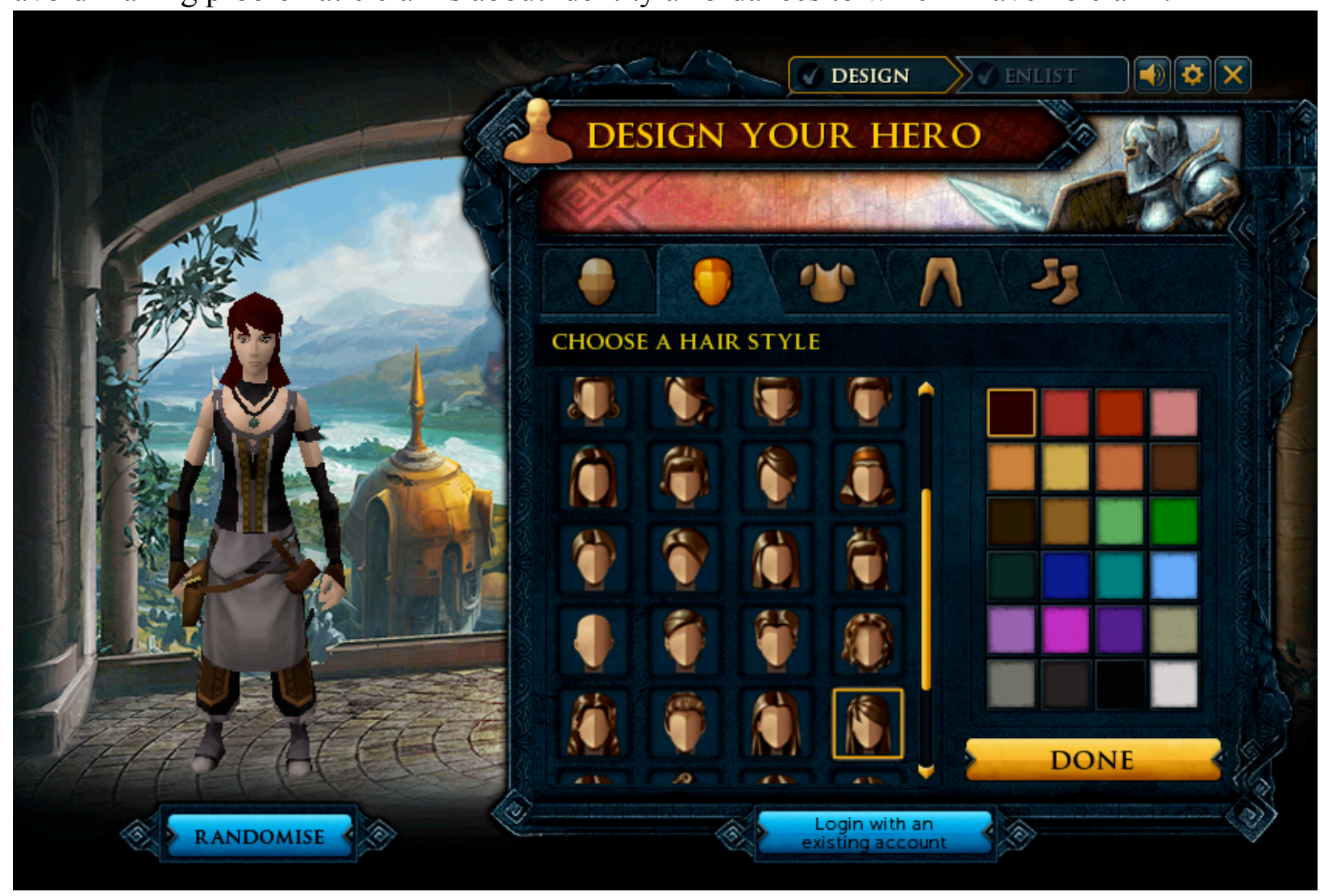

Figure 2: Creating my avatar in RuneScape 3. 


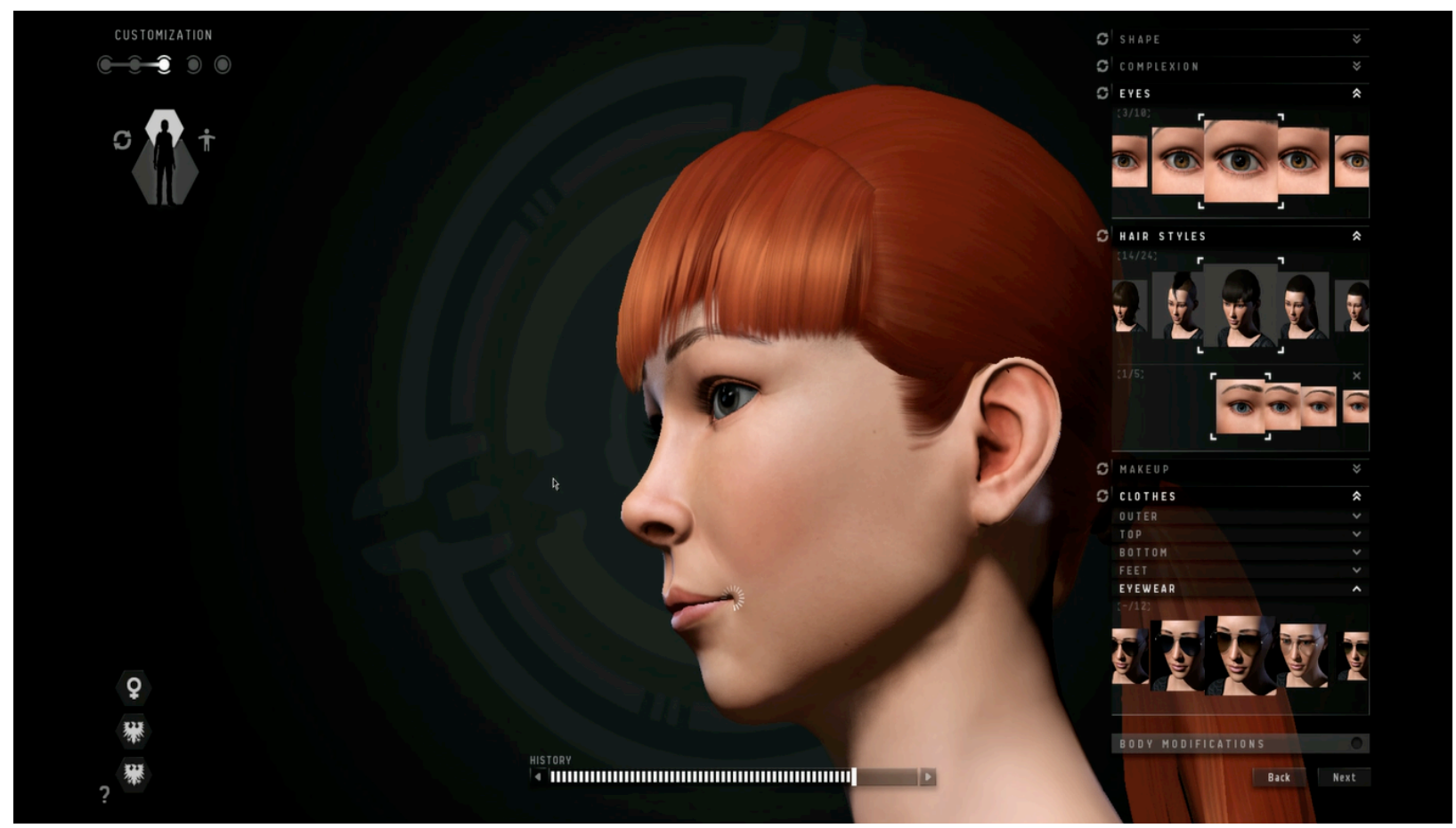

Figure 3: Creating my avatar in EVE Online.

Each interface was chosen due to my own familiarity and past experiences with these games. In all cases, I had spent a year or more playing with avatars in each of these ludic spaces. Notably, the interfaces vary with regard to complexity. For example, the Mii Creator and RuneScape 3 interfaces are both relatively low-fidelity affordances. In contrast, Saints Row IV, Skyrim, and EVE Online offer relatively high fidelity CCIs and produce more realistic looking avatars.

Although one might hypothesize that a higher fidelity or more complex interface would make it easier to create a representative avatar, this was not always the case. For example, in my detailed analysis of the avatar customization task in Skyrim, I spent a great deal of time accessing the individual widgets that allow players to painstakingly adjust the size and position of each facial feature discretely. In both EVE Online and Saints Row IV, I take advantage of similar affordances and am pleased with the result. However, I find it more difficult to create a representative avatar in Skyrim:

"Despite the affordances of the avatar creation interface, the pre-designed range of these affordances are set by the game's aesthetic. The avatars are designed to look rugged and battle-worn - to visually align with the game. This first becomes apparent in attempting to select my avatar's hairstyle - there are several options befitting of a Nordic warrior woman, but no long-ish hairstyles with bangs. I find many of the hairstyles appealing - the dreadlocks, for example, which I chose for my first Nord years ago, represent a spontaneous choice, granted by the game's aesthetics while not necessarily aligning with trends I have noted in other avatars I have created in other games... Here, the features seem to work against each other, rather than combining to represent my face." 
This exercise not only provided me, an ANT researcher, with the opportunity to present my own biases and experiences in a methodologically grounded way, but can also be synthesized to reveal a number of themes which not only had an impact on my own self-representational practices, but are also evident in how I approach identity work as a researcher. In total, I have produced four themes from this data: the self, affordances, aesthetics, and co-situated play.

The self: Player avatars are often constructed/theorized in relation to the self; a user-avatar dichotomy. Although an ANT approach results in a flat reading of the actor-network, the self is still very active within this network. I have not removed the self from the study of identity, rather, I have suggested that we consider other actors in the network as performing a kind of identity negotiation with users during avatar customization. The self is ever-present, making selections based on their desires or discoveries, but we should disrupt the thinking that has promoted a user-avatar dichotomy in the research. Similarly, games scholars need to make themselves visible in the theories they put forth - there is power in deciding what to weave into theory and a great deal of agency in our methodological and analytical choices.

Affordances: Affordances are the action possibilities of a game mechanic or interface. In designing and developing a game, the game designer has (intentionally or not) made choices about what we can do and how we should do it. The actual and perceived affordances have an impact on player avatar customization. Customization strategies often shift in-situ when new affordances are discovered. We do a disservice to identity theory when we evaluate made avatars as data.

Aesthetics: The range of customization options are often confined to those which are predetermined to "belong" to a predesigned game world. These aesthetics extend to avatar appearance and avatar bodies, where player avatars are expected to conform to the ideologies of that game world. Game worlds are not created in a vacuum, and there are few examples of game worlds that do not force users to adopt hypersexualized avatars that reflect a very rigid understanding of gendered bodies and self-expression. Therefore, aesthetics can and should make space for more diverse avatar bodies.

Co-situated play: The desire to play with one's peers may potentially limit selfrepresentation/strategies where the game splits customization options up between one or more faction. Co-situatedness may also have an impact on self-representation where one user's choice to create a representative avatar (or not) may inspire other co-situated players to do the same.

\section{Discussion, Conclusions, and Future Work}

In this paper, I presented a micro-autoethnographical study in which I attempted to create the same, self-representative avatar in eight different games. I chose to create what Pearce refers to as trans-ludic avatars (Pearce \& Artemesia, 2008); avatars that are customized by the user such 
that the same avatar appears to cross ludic boundaries. As Pearce notes (Pearce \& Artemesia, 2009a), the ability to create a trans-ludic avatar is a negotiation between user and interface affordances. As such, these avatars are often approximate simulacra - the result of a likeness which has been made to fit within a multitude of virtual aesthetics. This is illustrated in my own trans-ludic avatars, who are all made from me, but are not identical. Their differences are the result of differing affordances and game world aesthetics. The differences between each interface are systematically narrativised and analyzed via this micro-autoethnographic exercise.

I opted to include a micro-autoethnographical approach, using Pearce's trans-ludic identity as a procedural framework, not only as a means to illustrate the effects of affordances on analogous avatars created across multiple games, but also as a way to insert myself, including my own assumptions, biases, and experiences, into this research. As in all research that is theoretically framed via Actor-Network Theory, the researcher is a node in the network whose influence on the resultant theories must be disclosed and made visible. This paper also served to situate my own personal experiences and observations into this dissertation in a way that allows it to coexist with related data.

My own choice to create a representative (white) avatar does present a limitation of the research, given that my investigation into CCI affordances is informed by my interest in social inclusion, not usability. This decision was informed by Nakamura's (2002) discussions on identity tourism online, in which she describes the problematic affordance offered by virtual spaces, allowing one to appropriate another culture or identity online. Furthermore, this choice aligns with the primary impetus of this work - to insert myself, the researcher, squarely within my study and to make visible the ways in which my experiences within these environments has shaped my own research. I envision this method being taken up as a complementary approach to identity study in games where avatar customization is being studied. Further, the exercise may be useful as an instructional tool in game design programs in order to guide students in making informed choices about CCI design.

In future work, I intend to extend this method to a lab-based study involving a diverse participant pool, especially women, minorities, and non-binary folk. The impetus for this paper was to get the method out there - next, we need to mobilize it in order to better understand how marginalized players are truly impacted by CCIs.

\section{References}

Bergstrom, K., Jenson, J., \& de Castell, S. (2012). What's 'choice' got to do with it?: Avatar selection differences between novice and expert players of world of warcraft and Rift. Paper presented at the International Conference on the Foundations of Digital Games.

Bessière, K., Seay, A. F., \& Kiesler, S. (2007). The ideal elf: Identity exploration in world of warcraft. CyberPsychology and Behaviour, 10(4), 530-535. 
Boellstorff, T., Nardi, B., Pearce, C., \& Taylor, T. L. (2012). Ethnography and virtual worlds: A handbook of method: Princeton University Press.

Bogost, I. (2010). Persuasive games: The expressive power of videogames. Cambridge, Mass.: MIT Press.

Callon, M. (1997). Representing nature. Representing culture. Paris: CSI, Ecole Nationale Superieures des Mines.

Chen, M. (2009). Leet noobs: The life and death of an expert player group in world of warcraft. New York: Open University Press.

Consalvo, M. (2003a). Hot dates and fairy-tale romances: Studying sexuality in video games. In M. J. P. Wolf \& B. Perron (Eds.), The video game theory reader (pp. 171-194). London: Routledge.

Consalvo, M. (2003b). It's a queer world after all: Studying the Sims and sexuality. Glaad.

Dietrich, D. R. (2013). Avatars of whiteness: Racial expression in video game characters. Sociological Inquiry, 83(1), 82-105.

Downs, E., \& Smith, S. (2005). Keeping abreast of hypersexuality: A video game character analysis. Paper presented at the 55th Annual Conference on the International Association of Communication.

Ducheneaut, N., Wen, M.-H., Yee, N., \& Wadley, G. (2009). Body and mind: A study of avatar personalization in three virtual worlds. Paper presented at the Proceedings of the 27th international conference on Human factors in computing systems (CHI '09).

Ellingson, L. L., \& Ellis, C. (2008). Autoethnography as constructionist project. In J. A. Holstein \& J. F. Gubrium (Eds.), Handbook of constructionist research (pp. 445-466). New York: Guilford Press.

Ellis, C. (2004). The ethnography I: A methodological novel about autoethnography. Walnut Creek: AltaMira Press.

Ericsson, K., \& Simon, H. (1993). Protocol analysis: Verbal reports as data (2nd ed.). Boston, Mass.: MIT Press.

Giddings, S. (2009). Events and collusions: A glossary for the microethnography of video game play. Games and Culture, 4(2), 144-157.

Giddings, S., \& Kennedy, H. W. (2008). Little jesuses and*@\#?-off robots: On aesthetics, cybernetics, and not being very good at Lego Star Wars. In M. Swalwell, \& J. Wilson (Eds.), The pleasures of computer gaming: Essays on cultural history, theory and aesthetics. (pp. 13-32). Jefferson: NC: McFarland.

Higgin, T. (2009). Blackless fantasy: The disappearance of race in massively multiplayer online role-playing games. Games and Culture, 4(1), 3-26.

Higgin, T. (forthcoming). Technologics of race in videogames. In A. Brock (Ed.), The intersectional internet: Race, gender, and culture Online. Peter Lang.

Jin, S.-A. A. (2009). Avatars mirroring the actual self versus projecting the ideal self: The effects of self-priming on interactivity and immersion in an exergame, Wii Fit. CyberPsychology, Behaviour, and Social Networking, 12(6), 761 - 765.

Jin, S.-A. A. (2010). I feel more connected to the physically ideal mini me than the mirror-image mini me: Theoretical implications of the "malleable self" for speculations on the effects of avatar creation on avatar-self connection in Wii. CyberPsychology, Behaviour, and Social Networking, 13(5), 567 - 570. 
Kafai, Y. B., Fields, D. A., \& Cook, M. S. (2010a). 'Blacks deserve bodies too!': Design and discussion about diversity and race in a tween virtual world. Games and Culture, 5(1), 43-63.

Kafai, Y. B., Fields, D. A., \& Cook, M. S. (2010b). Your second selves: Player-designed avatars. Games and Culture, 5(1), 23-42.

Kolko, B. E. (1999). Representing bodies in virtual space: The rhetoric of avatar design. The Information Society, 15(3), 177-186.

Latour, B. (1987). Science in action: How to follow scientists and engineers through society. Milton Keynes: Open University Press.

Latour, B. (1992). Where are the missing masses? Sociology of a few mundane artefacts. In W. Bijker \& J. Law (Eds.), Shaping technology, building society: Studies in sociotechnical change. Cambridge, Mass: MIT Press.

Latour, B. (2005). Reassembling the social: An introduction to actor-network-theory. Oxford: Oxford University Press.

Law, J. (1992). Notes on the theory of the actor-network: Ordering, strategy, and heterogeneity. Systems practice, 5(4), 379-393.

Law, J. (2004). After method: Mess in social science research: Routledge.

Lawson, A., \& Murray, J. (2014). Identifying user demographic traits through virtual-world language use. In M. A. Ahmad, C. Shen, J. Srivastava \& N. Contractor (Eds.), Predicting real world behaviours from virtual world data (pp. 57-67): Springer.

Leonard, D. J. (2006). Not a hater, just keepin' it real: The importance of race- and gender-based game studies. Games and Culture, 1(1), 83-88.

Lewis, C. H. (1982). Using the "thinking aloud" method in cognitive interface design: IBM.

Maréchal, G. (2010). Autoethnography. In G. Durepos \& E. Wiebe (Eds.), Encyclopedia of case study research Vol. 2 (pp. 43-45). Thousand Oaks, CA: Sage Publications.

McArthur, V., \& Jenson, J. (2015). Plans and co-situated factions: An evaluation of avatar affordances in Rift's character creation interface. Journal of Gaming and Virtual Worlds, $7(1)$.

McArthur, V., Jenson, J., \& Teather, R. J. (2015). The avatar affordances framework: Mapping affordances and design trends in character creation interfaces. Paper presented at the ACM Symposium on Computer-Human Interaction in Play - CHI Play.

Nakamura, L. (2002). Cybertypes: Race, ethnicity, and identity on the internet. New York: Routledge.

Nardi, B. (2010). My life as a night elf priest: An anthropological account of world of warcraft: University of Michigan Press.

Neustaedter, C., \& Fedorovskaya, E. (2008). Establishing and maintaining relationships in a social virtual world. Eastman Kodak Company Technical Report 344195F.

Neustaedter, C., \& Fedorovskaya, E. (2009). Presenting identity in a virtual world through avatar appearances. Paper presented at the Proceedings of Graphics Interface 2009.

Pace, T. (2008). Can an orc catch a cab in stormwind?: Cybertype preference in the world of warcraft character creation interface. Paper presented at the CHI EA '08 CHI '08 Extended Abstracts on Human Factors in Computing Systems.

Pace, T., Houssian, A., \& McArthur, V. (2009). Are socially exclusive values embedded in avatar creation interfaces of MMORPGs? Journal of Information, Communication, and Ethics in Society, 7(2/3), 192-210. 
Pearce, C., \& Artemesia (2008). Identity-as-place: Trans-ludic identities in mediated play communities-the case of the Uru diaspora. Paper presented at the Internet 9.0: Association of Internet Researchers (AOIR).

Pearce, C., \& Artemesia (2009a). Communities of play: Emergent cultures in multiplayer games and virtual worlds. Cambridge, Mass.: MIT Press.

Pearce, C., \& Artemesia (2009b). Methodology: Playing ethnography. In Communities of play: Emergent cultures in multiplayer games and virtual worlds. Cambridge, Mass.: MIT Press.

Reed-Danahay, D. E. (1997). Auto/ethnography. New York: Berg.

Salen, K., \& Zimmerman, E. (2004). Rules of play: Game design fundamentals. Cambridge, Mass: MIT Press.

Smith, L. M., \& Geoffrey, W. (1968). The complexities of an urban classroom: An analysis toward a general theory of teaching. New York: Holt, Rinehart \& Winston.

Spry, T. (2001). Performing autoethnography: An embodied methodological praxis. Qualitative Inquiry, 7(6), 706-732.

Streeck, J., \& Mehus, S. (2005). Microethnography: The study of practices. In K. L. Fitch \& R. E. Sanders (Eds.), Handbook of language and social interaction. Mahwah, N.J.: Lawrence Erlbaum Associate.

Sundén, J. (2003). Material virtualities: Approaching online textual embodiment. New York: Peter Lang Publishing.

Sundén, J. (2009). Play as transgression: An ethnographic approach to queer game culture. Paper presented at DiGRA 2009.

Taylor, N. (2008). Periscopic play: Re-positioning 'the field' in MMO research. Loading... 1(3).

Taylor, N., McArthur, V., \& Jenson, J. (2012). Virtual postcards: Multimodal stories of online play. Paper presented at the CHI '12 Extended Abstracts on Human Factors in Computing Systems.

Taylor, T. L. (2006). Play between worlds: Exploring online game culture. Cambridge, Mass.: MIT Press. 